\title{
THE NETWORK PROBABILITY MODEL OF ONE-TYPE CUSTOMER REQUESTS PROCESSING IN AN INSURANCE COMPANY
}

\author{
Mikhail Matalytski ${ }^{1}$, Tatiana Rusilko ${ }^{2}$ \\ ${ }^{I}$ Institute of Mathematics, Czestochowa University of Technology, Poland \\ ${ }^{2}$ Grodno State University, Belarus \\ m.matalytski@gmail.com,romaniuk@grsu.by
}

\begin{abstract}
The subject of this paper is the stochastic model of client requests processed by an insurance company. The model takes into account the limited duration of insurance contracts and the dependence on time of requests service rate. A closed exponential queueing network with single-type messages is used as the model. The goal of the study is to solve the problem of finding the optimal number of employees of the insurance company on certain time intervals. The study is conducted in the asymptotic case of high network load. The results of this paper could be applied to optimize the functioning of insurance companies.
\end{abstract}

\section{Introduction}

The process of functioning of an insurance company, concluding same type insurance contracts with its clients is considered [1]. It's supposed that the maximum number of clients is $K$. For instance, it could be the citizenship of a town in which the company operates. $m_{4}$ of company employees engaged in contracting (insurers). Upon presentation of a claim, it goes through two stages of processing - the assessment stage and payment stage. The assessment of claims involved $m_{1}$ employees (evaluators). The payment of the charges involved $m_{3}$ lawsuits cashiers. Each of the company's customers can be in one of the following states: $C_{2}$ - in a waiting state, not going to submit an insurance claim; $C_{1}$ - in an assessment claim state; $C_{3}$ - in the cash transactions state; $C_{4}$ - in the state of making of a contract. Let's also introduce state $C_{0}$, meaning the staying of the company's potential customer in the "external environment". Assume that processing time of claims by evaluators is distributed exponentially with time-depending parameter $\mu_{1}(t)$, the processing time of customers by cashiers is exponentially distributed with $\mu_{3}(t)$, the processing time by insurers is exponentially distributed with $\mu_{4}(t)$.

The transition of some insurance claim from state $C_{0}$ to state $C_{4}$, as well as from $C_{2}$ to $C_{i}$, occurs at random instants of time independently on state of other claims, and regardless of the time so that probability of transition $C_{0} \longrightarrow C_{4}$ on 
time interval $[t, t+\Delta t]$ equals $\mu_{0}(t) \Delta t+o(t)$, and probability of transition $C_{2} \longrightarrow C_{i}$ on same time interval equals $\mu_{2 i}(t) \Delta t+o(t)$. Here $\mu_{0}(t)$, $\mu_{2 i}(t)=\mu_{2}(t) p_{2 i}(t)$ - are transition rates, because of seasonality of insurance processes it's convenient to represent rates as periodic or piecewise constant functions of time; $p_{2 i}(t)$ - time-dependent probabilities of transition from state $C_{2}$ to $C_{i}$, $0 \leq p_{2 i}(t) \leq 1, i=\overline{0,1}, p_{20}(t)+p_{21}(t)=1$.

The closed queueing network (Fig. 1) with $K$ messages circulating in it, which consists of five queueing systems $S_{0}, S_{1}, S_{2}, S_{3}, S_{4}$ could be used as a model of the processing of insurance claims processing described above, all queueing systems consist of $K, m_{1}, K, m_{3}$ and $m_{4}$ service lines accordingly [2]. The probabilities of messages (clients) transition between queueing systems are $p_{04}=p_{43}=p_{32}=p_{13}=1, p_{2 i}(t) \neq 0, i=\overline{0,1}$, in other cases $p_{i j}=0$. Service disciplines of messages by queueing systems are FIFO.

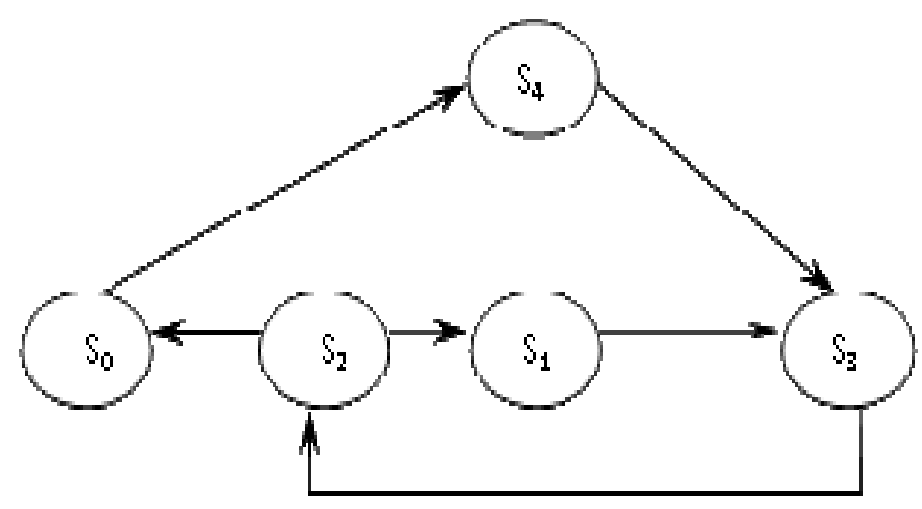

Fig. 1. Network structure

\section{Problem definition}

The state of the insurance company at time instant $t$ could be described by vector

$$
k(t)=(k, t)=\left(k_{1}(t), k_{2}(t), k_{3}(t), k_{4}(t)\right),
$$

where $k_{i}(t)$ - total number of messages that are on stage $C_{i}, i=\overline{1,4}$, and $k_{0}(t)=K-\sum_{i=1}^{4} k_{i}(t)$ - total number of messages on stage $C_{0}$.

Let's introduce the following cost factors:

$D_{2}$ - company's revenue per unit of time per customer, when the customer is not suing, i.e. message is on stage $C_{2}$; 
$D_{4}$ - company's revenue per unit of time per customer, when the customer is going to sign a contract, i.e. on stage $C_{4}$ (the amount of the premium the insurer introducing is taking into account);

$D_{1}$ - company's losses per unit of time per customer that is on stage of evaluating of the claim, i.e. on stage $C_{1}$ (the amount of the insurance paid and the cost estimate of the claim is taking into account);

$D_{3}$ - company's losses per unit of time from a single claim, when it is on the payment stage $C_{3}$ (the cost of customer service at the payment stage taking into account);

$E_{1}$ - the cost of keeping one evaluator per unit of time;

$E_{3}$ - the cost of maintaining a cashier per unit of time;

$E_{4}$ - the cost of keeping one insurer per unit of time.

Then the company's earnings at time $t$ is given by

$$
\Pi(t)=D_{2} k_{2}(t)+D_{4} k_{4}(t)-D_{1} k_{1}(t)-D_{3} k_{3}(t)-E_{1} m_{1}-E_{3} m_{3}-E_{4} m_{4} .
$$

Obviously $k(t)$ is the Markov process with continuous time and a finite set of states, so $\Pi(t)$ is also a random process. With $\Pi(t)$ it's easy to find an expression for the average income brought in by one customer at a time interval $\left[T_{1}, T_{2}\right]$ :

$$
\begin{gathered}
R\left(T_{1}, T_{2}, m_{1}, m_{3}, m_{4}\right)=\frac{1}{T_{2}-T_{1}} \int_{T_{1}}^{T_{2}} M\left\{\frac{\Pi(t)}{K}\right\} d t= \\
=\frac{1}{T_{2}-T_{1}} \int_{T_{1}}^{T_{2}}\left(\sum_{i=1}^{4} d_{i} n_{i}(t)-\sum_{\substack{i=1 \\
i \neq 2}}^{4} E_{i} l_{i}\right) d t,
\end{gathered}
$$

where $n_{i}(t)=M\left\{\frac{k_{i}(t)}{K}\right\}$ - the average relative number of customers on stage $C_{i}$, $i=\overline{1,4}, d_{1}=-D_{1}, d_{2}=D_{2}, d_{3}=-D_{3}, d_{4}=D_{4}, l_{i}=\frac{m_{i}}{K}, i=1,3,4$.

We are interested in the problem of determining the number of evaluators $m_{1}$, cashiers $m_{3}$ and insurers $m_{4}$ on the time interval $\left[T_{1}, T_{2}\right]$, that will maximize the average relative income (2) in the average absence of queues at the stages of customer service:

$$
\left\{\begin{array}{l}
W\left(T_{1}, T_{2}, m_{1}, m_{3}, m_{4}\right) \longrightarrow \max _{m_{1}, m_{3}, m_{4}}, \\
n_{i}(t) \leq l_{i}, i=1,3,4, t \in\left[T_{1}, T_{2}\right] .
\end{array}\right.
$$


To solve (3) it's necessary, first of all, to find the components of vector

$$
n(t)=\left(n_{1}(t), n_{2}(t), n_{3}(t), n_{4}(t)\right)
$$

\section{Obtaining the system of differential equations for the mean relative number of messages in queueing systems}

The following transitions into state $k(t+\Delta t)=(k, t+\Delta t)$ of the considered network during time $\Delta t$ are possible:

- from state $\left(k-I_{4}, t\right)$ with probability

$$
\mu_{0}(t)\left(k_{0}(t)+1\right) \Delta t+o(\Delta t)=\mu_{0}(t)\left(K-\sum_{i=1}^{4} k_{i}(t)+1\right) \Delta t+o(\Delta t)
$$

- from state $\left(k+I_{4}-I_{3}, t\right)$ with probability

$$
\mu_{4}(t) \min \left(m_{4}, k_{4}(t)+1\right) \Delta t+o(\Delta t) ;
$$

- from state $\left(k+I_{3}-I_{2}, t\right)$ with probability

$$
\mu_{3}(t) \min \left(m_{3}, k_{3}(t)+1\right) \Delta t+o(\Delta t) ;
$$

- from state $\left(k+I_{2}, t\right)$ with probability

$$
\mu_{2}(t) p_{20}(t)\left(k_{2}(t)+1\right) \Delta t+o(\Delta t) ;
$$

- from state $\left(k+I_{2}-I_{1}, t\right)$ with probability

$$
\mu_{2}(t) p_{21}(t)\left(k_{2}(t)+1\right) \Delta t+o(\Delta t) ;
$$

- from state $\left(k+I_{1}-I_{3}, t\right)$ with probability

$$
\mu_{1}(t) \min \left(m_{1}, k_{1}(t)+1\right) \Delta t+o(\Delta t) ;
$$

- from state $(k, t)$ with probability

$$
\begin{gathered}
1-\left[\mu_{0}(t)\left(K-\sum_{i=1}^{4} k_{i}(t)\right)+\sum_{\substack{i=1 \\
i \neq 2}}^{4} \mu_{i}(t) \min \left(m_{i}, k_{i}(t)\right)\right] \Delta t+ \\
+\mu_{2}(t) k_{2}(t) \Delta t+o(\Delta t) ;
\end{gathered}
$$

- from all other states with probability $o(\Delta t)$. 
Using the law of total probability and passing to the limit $\Delta t \rightarrow 0$, one can obtain the Kolmogorov system of difference-differentials equations for states probabilities

$$
\begin{gathered}
\frac{d P(k, t)}{d t}=\mu_{0}(t)\left(K-\sum_{i=1}^{4} k_{i}(t)\right)\left[P\left(k-I_{4}, t\right)-P(k, t)\right]+ \\
+\mu_{0}(t) P\left(k-I_{4}, t\right)+\mu_{4}(t) \min \left(m_{4}, k_{4}(t)\right)\left[P\left(k+I_{4}-I_{3}, t\right)-P(k, t)\right]+ \\
+\mu_{4}(t)\left[\min \left(m_{4}, k_{4}(t)+1\right)-\min \left(m_{4}, k_{4}(t)\right)\right] P\left(k+I_{4}-I_{3}, t\right)+ \\
+\mu_{3}(t) \min \left(m_{3}, k_{3}(t)\right)\left[P\left(k+I_{3}-I_{2}, t\right)-P(k, t)\right]+ \\
+\mu_{3}(t)\left[\min \left(m_{3}, k_{3}(t)+1\right)-\min \left(m_{3}, k_{3}(t)\right)\right] P\left(k+I_{3}-I_{2}, t\right)+ \\
+\mu_{2}(t) p_{20}(t) k_{2}(t)\left[P\left(k+I_{2}, t\right)-P(k, t)\right]+\mu_{2}(t) p_{20}(t) k_{2}(t) P\left(k+I_{2}, t\right)+ \\
+\mu_{2}(t) p_{21}(t) k_{2}(t)\left[P\left(k+I_{2}-I_{1}, t\right)-P(k, t)\right]+ \\
+\mu_{2}(t) p_{21}(t) k_{2}(t) P\left(k+I_{2}-I_{1}, t\right)+ \\
+\mu_{1}(t) \min \left(m_{1}, k_{1}(t)\right)\left[P\left(k+I_{1}-I_{3}, t\right)-P(k, t)\right]+ \\
+\mu_{1}(t)\left[\min \left(m_{1}, k_{1}(t)+1\right)-\min \left(m_{1}, k_{1}(t)\right)\right] P\left(k+I_{1}-I_{3}, t\right) .
\end{gathered}
$$

Next let's consider the case of a large number of messages in the network, $K>>1$, and introduce a vector of relative variables $\xi(t)=\left(\frac{k(t)}{K}\right)$, it's possible the values belong to a bounded closed set

$$
G=\left\{x=\left(x_{1}, x_{2}, x_{3}, x_{4}\right): x_{i} \geq 0, i=\overline{1,4}, \sum_{i=1}^{4} x_{i} \leq 1\right\},
$$

where they are placed in nodes of 4-dimensional lattice at a distance $\varepsilon=\frac{1}{K}$ from each other. By increasing $K$ "fill density" of set $G$ by possible components of this vector is increasing as well, and it becomes possible to assume that is has continuous distribution with probability density $p(x, t)=K^{4} P(x K, t), x \in G$, where $p(x, t)$ is the meaning of the probability density of the random vector $\xi(t)$.

Let's denote by $e_{i}$ 4-dimensional zero vector with the exception of $i$-th component that equals $\quad \varepsilon, \quad i=\overline{1,4}, \quad c(u)=\left\{\begin{array}{l}1, u>0, \\ 0, u \leq 0\end{array} . \quad\right.$ Here $\min (u, v+1)=\min (u, v)+c(u-v), \quad c(u-v)=\frac{\partial \min (u, v)}{\partial v}, \quad$ because $\quad$ of $\min (u, v)=\left\{\begin{array}{l}v, u \geq v, \\ u, u<v\end{array}\right.$. Rewriting system (5) for density $p(x, t)$, one get 


$$
\begin{gathered}
\frac{\partial p(x, t)}{\partial t}=K \mu_{0}(t)\left(1-\sum_{i=1}^{4} x_{i}\right)\left[p\left(x-e_{4}, t\right)-p(x, t)\right]+ \\
+\mu_{0}(t) p\left(x-e_{4}, t\right)+ \\
+K \mu_{4}(t) \min \left(l_{4}, x_{4}\right)\left[p\left(x+e_{4}-e_{3}, t\right)-p(x, t)\right]+ \\
+\mu_{4}(t) \frac{\partial \min \left(l_{4}, x_{4}\right)}{\partial x_{4}} p\left(x+e_{4}-e_{3}, t\right)+ \\
+K \mu_{3}(t) \min \left(l_{3}, x_{3}\right)\left[p\left(x+e_{3}-e_{2}, t\right)-p(x, t)\right]+ \\
+\mu_{3}(t) \frac{\partial \min \left(l_{3}, x_{3}\right)}{\partial x_{3}} p\left(x+e_{3}-e_{2}, t\right)+ \\
+K \mu_{2}(t) p_{20}(t) x_{2}\left[p\left(x+e_{2}, t\right)-p(x, t)\right]+ \\
+\mu_{2}(t) p_{20}(t) p\left(x+e_{2}, t\right)+ \\
+K \mu_{2}(t) p_{21}(t) x_{2}\left[p\left(x+e_{2}-e_{1}, t\right)-p(x, t)\right]+ \\
+\mu_{2}(t) p_{21}(t) p\left(x+e_{2}-e_{1}, t\right)+ \\
+K \mu_{1}(t) \min \left(l_{1}, x_{1}\right)\left[p\left(x+e_{1}-e_{3}, t\right)-p(x, t)\right]+ \\
+\mu_{1}(t) \frac{\partial \min \left(l_{1}, x_{1}\right)}{\partial x_{1}} p\left(x+e_{1}-e_{3}, t\right) .
\end{gathered}
$$

Let's represent the right-hand side of this system of equations up to terms of order of smallness $\varepsilon^{2}$. If $p(x, t)$ is twice differentiable by $x$, then the relations:

$$
\begin{gathered}
p\left(x \pm e_{i}, t\right)=p(x, t) \pm \varepsilon \frac{\partial p(x, t)}{\partial x_{i}}+\frac{\varepsilon^{2}}{2} \frac{\partial^{2} p(x, t)}{\partial x_{i}^{2}}+o\left(\varepsilon^{2}\right), \\
p\left(x+e_{i}-e_{j}, t\right)=p(x, t)+\varepsilon\left(\frac{\partial p(x, t)}{\partial x_{i}}-\frac{\partial p(x, t)}{\partial x_{j}}\right)+ \\
+\frac{\varepsilon^{2}}{2}\left(\frac{\partial^{2} p(x, t)}{\partial x_{i}^{2}}-2 \frac{\partial^{2} p(x, t)}{\partial x_{i} \partial x_{j}}+\frac{\partial^{2} p(x, t)}{\partial x_{j}^{2}}\right)+o\left(\varepsilon^{2}\right), \\
i, j=\overline{1,4} .
\end{gathered}
$$

Using it and also $\varepsilon K=1$ one can obtain that the probability density function $p(x, t)$ of the network states vector satisfies the Fokker-Planck-Kolmogorov equation up to terms of order of smallness $\varepsilon^{2}$ :

$$
\frac{\partial p(x, t)}{\partial t}=-\sum_{i=1}^{4} \frac{\partial}{\partial x_{i}}\left(A_{i}(x, t) p(x, t)\right)+\frac{\varepsilon}{2} \sum_{i, j=1}^{4} \frac{\partial^{2}}{\partial x_{i} \partial x_{j}}\left(B_{i j}(x, t) p(x, t)\right),
$$


where

$$
\begin{gathered}
A_{1}(x, t)=\mu_{2}(t) p_{21}(t) x_{2}-\mu_{1}(t) \min \left(l_{1}, x_{1}\right) \\
A_{2}(x, t)=\mu_{3}(t) \min \left(l_{3}, x_{3}\right)-\mu_{2}(t) x_{2} ; \\
A_{3}(x, t)=\mu_{4}(t) \min \left(l_{4}, x_{4}\right)+\mu_{1}(t) \min \left(l_{1}, x_{1}\right)-\mu_{3}(t) \min \left(l_{3}, x_{3}\right) \\
A_{4}(x, t)=\mu_{0}(t)\left(1-\sum_{i=1}^{4} x_{i}\right)-\mu_{4}(t) \min \left(l_{4}, x_{4}\right) \\
B_{11}(x, t)=\mu_{2}(t) p_{21}(t) x_{2}+\mu_{1}(t) \min \left(l_{1}, x_{1}\right) ; \\
B_{22}(x, t)=\mu_{3}(t) \min \left(l_{3}, x_{3}\right)+\mu_{2}(t) x_{2} ; \\
B_{33}(x, t)=\mu_{3}(t) \min \left(l_{3}, x_{3}\right)+\mu_{4}(t) \min \left(l_{4}, x_{4}\right) \\
B_{44}(x, t)=\mu_{4}(t) \min \left(l_{4}, x_{4}\right)+\mu_{0}(t)\left(1-\sum_{i=1}^{4} x_{i}\right) \\
B_{12}(x, t)=B_{21}(x, t)=-\mu_{2}(t) p_{21}(t) x_{2} ; \\
B_{13}(x, t)=B_{31}(x, t)=-\mu_{1}(t) \min \left(l_{1}, x_{1}\right) \\
B_{23}(x, t)=B_{32}(x, t)=-\mu_{3}(t) \min \left(l_{3}, x_{3}\right) \\
B_{34}(x, t)=B_{43}(x, t)=-\mu_{4}(t) \min \left(l_{4}, x_{4}\right) \\
B_{14}(x, t)=B_{41}(x, t)=B_{24}(x, t)=B_{42}(x, t)=0 .
\end{gathered}
$$

Equation (6) is the Fokker-Planck-Kolmogorov equation for the probability density function of the Markov process $\xi(t)$. So components of vector of mean relative to the number of messages in queueing systems are $n(t)=\left(n_{1}(t), n_{2}(t), \ldots, n_{n+2}(t)\right)$, where $n_{i}(t)=M\left(\frac{k_{i}(t)}{K}\right), i=\overline{1,4}$. According to [3] these components satisfy the following system of ordinary differential equations in terms of order of smallness $O\left(\varepsilon^{2}\right)$ :

$$
n_{i}^{\prime}(t)=A_{i}(n(t)), i=\overline{1,4}
$$

or using (7), we obtain the following system:

$$
\left\{\begin{aligned}
n_{1}^{\prime}(t)= & \mu_{2}(t) p_{21}(t) n_{2}(t)-\mu_{1}(t) \min \left(l_{1}, n_{1}(t)\right) \\
n_{2}^{\prime}(t)= & \mu_{3}(t) \min \left(l_{3}, n_{3}(t)\right)-\mu_{2}(t) n_{2}(t) \\
n_{3}^{\prime}(t)= & \mu_{4}(t) \min \left(l_{4}, n_{4}(t)\right)+\mu_{1}(t) \min \left(l_{1}, n_{1}(t)\right)- \\
& \quad-\mu_{3}(t) \min \left(l_{3}, n_{3}(t)\right) \\
n_{4}^{\prime}(t)= & \mu_{0}(t)\left(1-\sum_{i=1}^{4} n_{i}(t)\right)-\mu_{4}(t) \min \left(l_{4}, n_{4}(t)\right) .
\end{aligned}\right.
$$


Right-hand sides of (9) are continuous piecewise linear functions. Such systems could be solved by splitting the phase space and finding solutions in the areas of linearity of the right-hand sides. For instance, in the area corresponding to the case of missed queues on client servicing stages $n_{i}(t) \leq l_{i}, i=\overline{1,4}$, system (9) has the form

$$
\left\{\begin{array}{l}
\frac{d n_{1}(t)}{d t}=\mu_{2}(t) p_{21}(t) n_{2}(t)-\mu_{1}(t) n_{1}(t), \\
\frac{d n_{2}(t)}{d t}=\mu_{3}(t) n_{3}(t)-\mu_{2}(t) n_{2}(t), \\
\frac{d n_{3}(t)}{d t}=\mu_{4}(t) n_{4}(t)+\mu_{1}(t) n_{1}(t)-\mu_{3}(t) n_{3}(t), \\
\frac{d n_{4}(t)}{d t}=\mu_{0}(t)\left(1-n_{1}(t)-n_{2}(t)-n_{3}(t)-n_{4}(t)\right)-\mu_{4}(t) n_{4}(t) .
\end{array}\right.
$$

Solving (10) under certain initial conditions, for example $n_{i}(0)=0$, we obtain $n_{i}(t), i=\overline{1,4}$, and we can begin to solve the problem (3). It should be noted that the analytic solution of $(10)$ in the case when $\mu_{i}(t)$ is a function of time, is difficult.

\section{The solution of the optimization problem}

Obviously the right-hand side of (10) doesn't contain $m_{i}, i=1,3,4$, therefore it's solutions $n_{i}(t), i=\overline{1,4}$, also do not depend on $m_{i}, i=1,3,4$. Then the objective function of problem (3) has the form $W\left(T_{1}, T_{2}, m_{1}, m_{3}, m_{4}\right)=$ $=f\left(T_{1}, T_{2}\right)-C_{1} m_{1}-C_{3} m_{3}-C_{4} m_{4}$, where $C_{i}$ - nonnegative constants, $i=1,3,4$, and the solution of problem (3) will be the smallest $m_{i}, i=1,3,4$, which satisfy the constraints of the optimization problem. That is the solution of (3) which has the form

$$
m_{1}^{\circ}=\left\langle N_{1}\right\rangle, m_{3}^{\circ}=\left\langle N_{3}\right\rangle, m_{4}^{\circ}=\left\langle N_{4}\right\rangle,
$$

where $N_{i}=K \max _{t \in\left[T_{1}, T_{2}\right]}\left(n_{i}(t)\right), i=1,3,4,\langle x\rangle=\left\{\begin{array}{l}x, x \in Z \\ {[x]+1, x \notin Z}\end{array},[x]-\right.$ integral part of $x, Z$ - the set of integers.

In practice, the service rate and probability $p_{21}(t)$ are often defined by piecewise constant functions of time, for example, with two intervals of constancy: 


$$
\mu_{i}(t)=\left\{\begin{array}{l}
\mu_{i}^{(1)}, t \in\left[T_{1}, T_{2} / 2\right], \\
\mu_{i}^{(2)}, t \in\left(T_{2} / 2, T_{2}\right] ;
\end{array} i=\overline{0,4}, p_{21}(t)=\left\{\begin{array}{l}
p_{21}^{(1)}, t \in\left[T_{1}, T_{2} / 2\right] \\
p_{21}^{(2)}, t \in\left(T_{2} / 2, T_{2}\right]
\end{array}\right.\right.
$$

Then system (10) is a system of linear differential equations with constant coefficients. And if all the eigenvalues of (10) have strictly negative real parts, then there are stationary solutions when $t \rightarrow+\infty$. In some cases, a steady state is achieved within a very short time interval. Therefore, when considering sufficiently large time intervals to solve the optimization problem, sometimes it's better to use the stationary solution of (10). Namely, the optimal number of employees should be determined by formulas (11) on each of the intervals of constancy (12). Moreover, on time interval $\left[T_{1}, T_{2} / 2\right]$ we need to consider that $N_{i}^{(1)}=K \lim _{t \rightarrow T_{2} / 2} n_{i}(t), \quad$ and $\quad$ on time interval $\left(T_{2} / 2, T_{2}\right]$ need assume $N_{i}^{(2)}=K \lim _{t \rightarrow T_{2}}\left(n_{i}(t)\right), i=1,3,4$.

\subsection{Example}

Let's assume $K=40000$. The functioning of the insurance company described by the following parameters:

$$
\begin{gathered}
\mu_{0}(t)=0.0007 \sin (2 \pi t / 364)+0.0008, \mu_{1}(t)=0.00005 \sin (2 \pi t / 364)+0.00008, \\
\mu_{2}(t)=0.0005 \cos (2 \pi t / 364)+0.006, \mu_{3}(t)=11 \sin (2 \pi t / 364)+20, \\
\mu_{4}(t)=3.5 \cos (2 \pi t / 364)+9, p_{21}(t)=0.004 \sin (2 \pi t / 364)+0.008
\end{gathered}
$$

We will investigate the company's work on the time interval $[0,364]$ with the initial condition $n_{i}(0)=0, i=\overline{1,4}$. Let's solve problem (3).

The right-hand side of (10) with this condition doesn't depend on $m_{i}, i=1,3,4$. Therefore, to solve the optimization problem of the insurance company it is sufficient to know the type of solutions of (10) in this case. Hence, for the solution of the system it's possible to use numerical methods. For the numerical solution of (10) mathematical computer software Maple could be applied. In particular, function dsolve with option type=numeric together with method-options which allow for the determination of a method of numerical solutions, and function odeplot for graphical representation of solution, could be used.

Figures 2-4 graphically represent the behavior of functions $K n_{i}(t), i=1,3,4$, - the average number of messages on stages of evaluation, payment and contracting under the above initial conditions. 


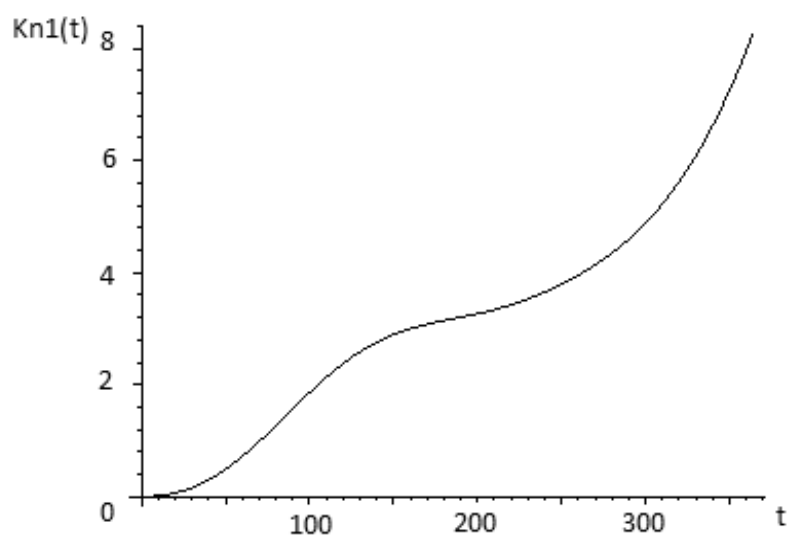

Fig. 2. Chart of $K n_{1}(t)$

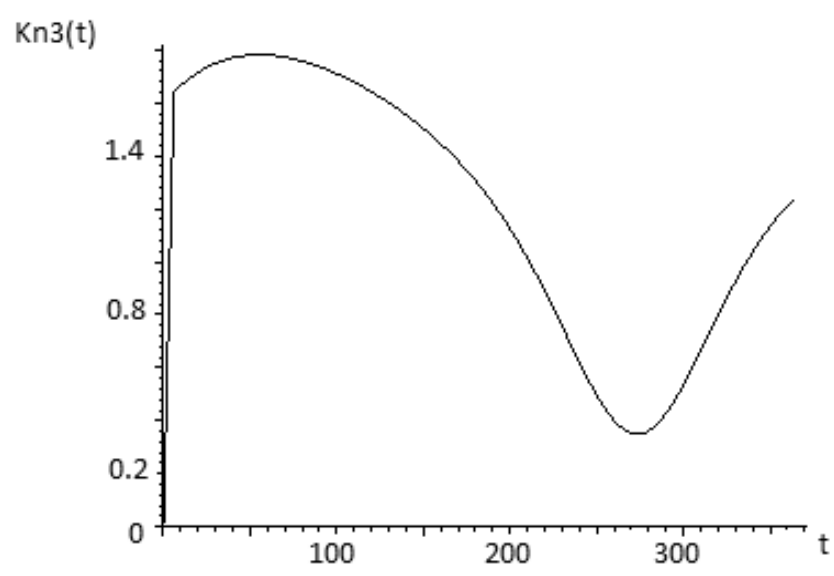

Fig. 3. Chart of $K n_{3}(t)$

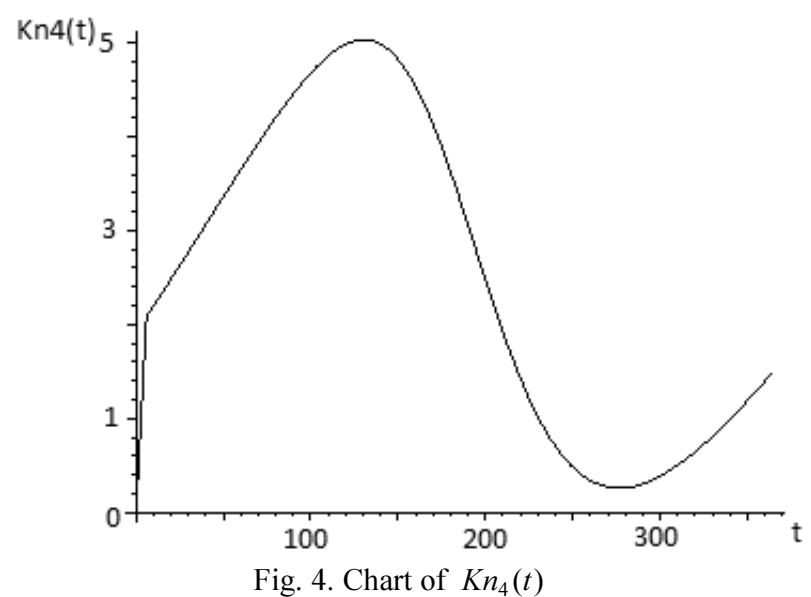


According to (11) we obtain $m_{1}^{\circ}=\langle 8.33\rangle=9, m_{3}^{\circ}=\langle 1.79\rangle=2, m_{4}^{\circ}=\langle 4.96\rangle=5$. Hence, the optimal number of evaluators -9 , cashiers -2 , insurers -5 .

\section{Conclusions}

These studies are valid only at a high network load, i.e. in case of large number $K$. The accuracy of results increases with the number of messages in the network. The procedure for the computer mathematical system Maple that makes it possible calculate examples was implemented. The results of this paper could be applied to optimize the process of functioning of insurance companies.

\section{References}

[1] Matalytski M., Rusilko T., Mathematical analysis of stochastic models of claims processing in insurance companies, GrSU, Grodno 2007.

[2] Rusilko T., Zagainova T., About one probabilistic model of clients processing in insurance company, Vestnik GrSU. 2008, 2 (3), 66-72.

[3] Paraev U., Introduction in Statistical Dynamics of Control and Filtration Processes, Sovetskoe radio, Moscow 1976. 